\title{
Association of perfluorinated chemical exposure in utero with maternal and infant thyroid hormone levels in the Sapporo cohort of Hokkaido Study on the Environment and Children's Health
}

\author{
Shizue Kato ${ }^{1}$ Sachiko Itoh ${ }^{2}$ Motoyuki Yuasa ${ }^{3}$ Toshiaki Baba $^{1}$. \\ Chihiro Miyashita $^{2} \cdot$ Seiko Sasaki $^{1} \cdot$ Sonomi Nakajima $^{4}$ Akiko Uno ${ }^{1}$. \\ Hiroyuki Nakazawa ${ }^{5} \cdot$ Yusuke Iwasaki $^{5} \cdot$ Emiko Okada $^{1} \cdot$ Reiko Kishi $^{2}$ \\ Received: 19 November 2015/Accepted: 13 April 2016/Published online: 30 April 2016 \\ (C) The Japanese Society for Hygiene 2016
}

\begin{abstract}
Objectives Perfluorooctane sulfonate (PFOS) and perfluorooctanoate (PFOA) have been widely used as industrial products, and are persistent organic pollutants due to their chemical stability. Previous studies suggested that PFOS and PFOA might disrupt thyroid hormone (TH) status. Although TH plays an important role in fetal growth during pregnancy, little attention has been paid to the relationships between maternal exposure to perfluorocarbons and $\mathrm{TH}$ statuses of mothers and fetuses. We investigated the effects of low levels of environmental PFOS and PFOA on thyroid function of mothers and infants.

Methods Of the eligible subjects in a prospective cohort, 392 mother-infant pairs were selected. Concentration of maternal serum PFOS and PFOA was measured in samples taken during the second and third trimesters or within 1 week of delivery. Blood samples for measuring thyroid
\end{abstract}

Electronic supplementary material The online version of this article (doi:10.1007/s12199-016-0534-2) contains supplementary material, which is available to authorized users.

Reiko Kishi

rkishi@med.hokudai.ac.jp

1 Department of Public Health, Hokkaido University Graduate School of Medicine, Sapporo, Japan

2 Center for Environmental and Health Sciences, Hokkaido University, Sapporo, Japan

3 Department of Public Health, School of Medicine, Juntendo University, Tokyo, Japan

4 Department of Occupational Therapy, School of Health Sciences, Sapporo Medical University, Sapporo, Japan

5 Department of Analytical Chemistry, School of Pharmacy and Pharmaceutical Sciences, Hoshi University, Tokyo, Japan stimulating hormone (TSH) and free thyroxine (FT4) levels were obtained from mothers at early gestational stage (median 11.1 weeks), and from infants between 4 and 7 days of age, respectively.

Results Median concentrations of PFOS and PFOA were 5.2 [95\% confidence interval (CI) 1.6-12.3] and 1.2 (95\% CI limitation of detection-3.4) ng/mL, respectively. Maternal PFOS levels were inversely correlated with maternal serum TSH and positively associated with infant serum TSH, whereas maternal PFOA showed no significant relationship with TSH or FT4 among mothers and infants.

Conclusions These findings suggest that PFOS may independently affect the secretion and balances of maternal and infant TSH even at low levels of environmental exposure.

Keywords Perfluorooctane sulfonate .

Perfluorooctanoate $\cdot$ Thyroid hormones $\cdot$ Mother-infant pairs $\cdot$ Prenatal exposure

\section{Introduction}

Perfluorinated compounds (PFCs) are synthetic chemicals that have been manufactured since the 1950s. Perfluorooctane sulfonate (PFOS) and perfluorooctanoate (PFOA) are the most common chemicals among PFCs, and are quite stable because of the extremely strong bond between carbon and fluorine atoms. This characteristic has made them useful as surfactants, water repellants, and a variety of other industrial products for more than half a century. Human exposure to PFCs mainly occurs orally, via the intake of contaminated food, water, and dust [1]. Their presence in human blood has been reported in several countries [2-5]. Recently, potential adverse effects of 
PFCs on human health have been claimed, and PFOS was added to Annex B of the Stockholm Convention on Persistent Organic Pollutants in 2009 due to potential adverse human health and environmental effects. Although PFOS and PFOA are being voluntarily phased out by several industries and substituted by short chain products, they are still present in older products and in human serum due to their long half-lives (5.3 years for PFOS and 3.8 years for PFOA) [6]. Additionally, maternal PFOS and PFOA are transferred to fetuses across the placental barrier in humans [7, 8]. Therefore, there is a significant concern over the adverse effects of in utero exposure to PFOS and PFOA.

Epidemiological studies have reported that in utero exposure to PFOS and PFOA had adverse effects on birth sizes and childhood neurodevelopment [9, 10]. We previously reported an inverse association between maternal PFOS serum levels and birth weight among female infants [11]. Moreover, we found inverse correlations between PFOS and essential fatty acid and triglyceride levels during pregnancy, at levels of PFOS and PFOA that were lower than those previously reported [12].

Some recent studies have suggested that PFCs may disrupt thyroid function. In rat dams and their pups, PFOS exposure during pregnancy has been reported to reduce concentrations of the thyroid hormones (THs) triiodothyronine (T3) and thyroxine (T4) of dams and pups due to the competitive PFOS binding to protein and increased clearance of THs, without affecting the stimulation of thyroid stimulating hormone (TSH) [13, 14]. Furthermore, human cross-sectional studies in pregnant women have also revealed associations between higher levels of PFCs and increased maternal TSH levels, along with decreased T3 and T4 levels [1517]. In addition, two prospective studies concluded that prenatal exposure to PFCs alters the balance of THs in infants [18, 19]. However, their sample sizes were relatively small.

Thyroid hormones are very important for fetal growth in utero. Fetuses depend on their mothers' supply of THs in early gestation; hence, disruption of maternal $\mathrm{TH}$ homeostasis during early pregnancy might impair fetal development. Mild maternal hypothyroxinemia during pregnancy increases the risk for adverse effects on fetal neurodevelopment. However, there have been no reports describing the effects of PFCs on both maternal TH at early gestational stage and infant THs. Therefore, the aim of this study was to examine potential associations between levels of maternal PFOS and PFOA, and THs, TSH, and free thyroxine (FT4) in mother-infant pairs.

\section{Materials and methods}

\section{Study population and study design}

This study was based on data from the Hokkaido Study on the Environment and Children's Health, which involved mothers and their infants delivered at the Sapporo Toho Hospital in Sapporo, Hokkaido, Japan [20]. In brief, pregnant women between 23 and 35 weeks of gestation were recruited between 2002 and 2005. Of 1796 potentially eligible pregnant women who visited the hospital for prenatal health care, 514 women $(29 \%)$ agreed to participate in the study. Among 514 women, we excluded 10 who did not maintain participation to the end of the study (7 voluntarily withdrew, 2 had a miscarriage or stillbirth, and 1 relocated), as well as 7 who had twins. For the analysis of associations between maternal PFC levels and maternal and infant TH levels, the following subjects were excluded: those undergoing treatment for thyroid disease $(n=14)$, those of whom maternal and/or infants' TH data were not available $(n=40)$, and those with no data for serum PFOS and PFOA levels $(n=51)$. Ultimately, 392 mother-infant pairs were included in this study. The analysis of mothers regarding the associations between maternal PFOS and PFOA levels and maternal thyroid status during pregnancy were designed as the cross-sectional study, and the analysis of infants regarding the associations between maternal PFOS and PFOA levels and infants' thyroid status at birth were designed as the prospective study.

\section{Data collection}

At enrolment, participants completed a self-administered questionnaire that included questions about relevant sociodemographic, medical, and behavioral information, as described previously [11, 21]. In brief, the questionnaire extracted information related to medical history, dietary habits, smoking status, alcohol intake, household income, and educational level. We also obtained information from medical records on maternal age, maternal height, maternal pre-pregnancy weight, parity, pregnancy complications, gestational age, infant gender, birth weight, and birth length. This study was conducted with written informed consent from all subjects and was approved by the ethics committees of Hokkaido University Graduate School of Medicine (28 October 2002).

\section{TH measurement}

Thyroid function and antithyroid antibody data of a mass screening test at the Sapporo City Institute of Public Health 
were obtained. The blood samples were collected from mothers between the 7th and 20th weeks of gestation and from infants between 4 and 7 days old, respectively. TSH and FT4 levels were measured using enzyme-linked immunosorbent assay (TSH: Enzaplate N-TSH, Bayer Co., Tokyo, Japan; FT4: Enzaplate N-FT4, Bayer Co.). The FT4 values of all samples were detected. For 85 samples with TSH levels below the detection limit $(0.50 \mu \mathrm{U} / \mathrm{mL})$, we used a value of half the detection limit $(0.25 \mu \mathrm{U} / \mathrm{mL})$. Antimicrosomal antibody (AMC) and antithyroglobulin antibody (ATG) were measured by indirect agglutination reactions (AMC: Serodia AMC, Fuji-Rebio, Tokyo, Japan; ATG: Serodia ATG, Fuji-Rebio). Values of AMC and ATG above 100-fold of baseline were classified as positive.

\section{Blood sampling and measurement of PFOS and PFOA levels in maternal serum}

We used the data of PFOS and PFOA concentrations in maternal serum measured in our previous study. Detailed methods for the measurement of PFOS and PFOA have been described previously [11,21]. Forty milliliters of blood were collected from the maternal peripheral vein between 24 and 41 weeks of gestational age or within 5 days after delivery, and were stored at $-80{ }^{\circ} \mathrm{C}$ until analysis. PFOS values of all samples were determined; for samples with PFOA levels below the detection limit $(0.50 \mathrm{ng} / \mathrm{mL})$, we used a value of half the detection limit $(0.25 \mathrm{ng} / \mathrm{mL})$.

\section{Data analysis}

The Mann-Whitney and Spearman correlation tests were conducted to examine associations between levels of PFOS and PFOA in maternal serum and characteristics of the subjects. Associations of maternal serum PFOS and PFOA levels with TSH and FT4 levels of mothers and infants were analyzed using Spearman's correlation test. We also performed multiple linear regression analysis; the PFOS and PFOA values as well as the TSH and FT4 values were converted to a $\log 10$ scale because they did not fall into a normal distribution. In addition, the relationships between quartile PFOS concentrations in maternal serum and TSH levels of mothers and infants with a $p$ value of $<0.10$ in adjusted models shown in multiple linear regression analysis were assessed to clarify the dose-response relationship between maternal serum PFCs and THs; PFOS and PFOA levels were divided into 4 quartiles, and TSH and FT4 levels were converted to a $\log 10$ scale. The least square means of TSH and FT4 were calculated and back-transformed, and the $p$ value for their trends determined. The first quartile was also compared to the second, third, and fourth quartiles of PFOS and PFOA, and $p$ values were calculated using the Hsu-Dunnett's test. Analyses were conducted fully adjusting for confounding variables that were found to be significantly associated with PFOS, PFOA, TSH, and FT4 levels in our results (those with $p<0.05$ in Table 3 and Supplemental Table 1) and in previous literatures. For maternal analyses, values were adjusted for maternal age at delivery (years), body mass index (BMI) before pregnancy $\left(\mathrm{kg} / \mathrm{m}^{2}\right)$, parity $(0 / \geq 1)$, educational level ( $\leq 12 / \geq 13$ years), positive for ATG and/ or AMC (yes/no), intake of seaweed more than once a week (yes/no), blood sampling period for PFOS and PFOA (before/after delivery), and the gestational week at which blood sampling for TSH and FT4 was performed (continuous variable). For infant analysis, we selected maternal confounding factors [maternal age at delivery (years), parity $(0 / \geq 1)$, positivity for ATG and/or AMC (yes/no), blood sampling period for PFOS and PFOA (before/after delivery), and $\log 10$ values of TSH and FT4 (continuous variable)], and infant factors [gestational weeks for birth (continuous variable), low birth weight $<2500 \mathrm{~g}$ (yes/no), and born via cesarean section (yes/no)].

All statistical analyses were performed using JMP Pro 12 (SAS Institute Inc., NC, USA). Results were considered statistically significant if the $p$ value was below 0.05 .

\section{Results}

Table 1 shows the comparison of maternal characteristics and the concentrations of PFOS, PFOA, TSH, and FT4 by infant sex. Among 392 mothers of total infants, the median concentrations of PFOS and PFOA were 5.2 [95\% confidence interval (CI) 1.6-12.3] and 1.2 [95\% CI limit of detection (LOD)-3.4] ng/mL, respectively. TSH levels from $307(78.3 \%)$ maternal blood samples were above the LOD. The median levels of maternal TSH and FT4 levels were $1.0 \mu \mathrm{U} / \mathrm{mL}$ (95\% CI LOD-4.1 $\mu \mathrm{U} / \mathrm{mL})$ and $0.99 \mathrm{ng} /$ $\mathrm{mL}(95 \%$ CI $0.62-1.67 \mathrm{ng} / \mathrm{mL})$, respectively. There was no significant difference of between maternal levels of PFOS, PFOA, TSH, and FT4, and other maternal characteristics between mothers of male infants and female infants. As for infants in Table 2, the values of TSH above the LOD were detected from $373(95.2 \%)$ of infant's blood samples. The median levels of infants' TSH and FT4 levels were $2.2 \mu \mathrm{U} / \mathrm{mL}$ (95\% CI LOD-7.9 $\mu \mathrm{U} / \mathrm{mL}$ ) and $2.03 \mathrm{ng} / \mathrm{mL}$ (95\% CI $1.39-2.89 \mathrm{ng} / \mathrm{mL}$ ), respectively. The percentage of female infants with low birth weight $(<2500 \mathrm{~g})$ was slightly higher than that of male infants, however, it was not significant. The correlations between maternal TSH and infants' FT4 were found to be significant inversely (Spearman's $\rho=-0.104, p=0.040$ ), while maternal TSH did not correlated with infants' TSH. The maternal serum PFOS and PFOA concentrations in 
Table 1 Comparisons of maternal characteristics and the concentrations of PFOS, PFOA, TSH, and FT4 by infant sex

\begin{tabular}{|c|c|c|c|c|}
\hline Characteristics of mothers & $\begin{array}{l}\text { Mothers of total } \\
\text { infants }(n=392) \\
\text { Median } \pm \text { SD or } n(\%)\end{array}$ & $\begin{array}{l}\text { Mothers of male } \\
\text { infants }(n=180) \\
\text { Median } \pm \text { SD or } n(\%)\end{array}$ & $\begin{array}{l}\text { Mothers of female } \\
\text { infants }(n=212) \\
\text { Median } \pm \text { SD or } n(\%)\end{array}$ & $p$ value $^{\mathrm{a}}$ \\
\hline Age (years) at delivery & $31.1 \pm 7.1$ & $31.4 \pm 7.2$ & $30.8 \pm 7.1$ & 0.373 \\
\hline BMI $\left(\mathrm{kg} / \mathrm{m}^{2}\right)$ before pregnancy & $21.3 \pm 3.3$ & $21.2 \pm 3.2$ & $21.3 \pm 3.3$ & 0.678 \\
\hline \multicolumn{5}{|l|}{ Parity (times) } \\
\hline 0 & $176(44.9)$ & $85(47.2)$ & $91(42.9)$ & \multirow[t]{2}{*}{0.384} \\
\hline$\geq 1$ & $212(54.1)$ & $93(51.6)$ & $119(56.1)$ & \\
\hline \multicolumn{5}{|l|}{ Educational level (years) } \\
\hline$\leq 12$ & $178(45.4)$ & $83(46.1)$ & $95(44.8)$ & \multirow[t]{2}{*}{0.797} \\
\hline$\geq 13$ & $214(54.6)$ & $97(53.9)$ & $117(55.2)$ & \\
\hline \multicolumn{5}{|l|}{ Annual income (millions of yens) } \\
\hline$<5$ & $269(68.6)$ & $121(67.2)$ & $148(69.8)$ & \multirow[t]{2}{*}{0.645} \\
\hline$\geq 5$ & $120(30.6)$ & $57(31.7)$ & $63(29.7)$ & \\
\hline \multicolumn{5}{|l|}{ Smoking during pregnancy } \\
\hline Yes & $67(17.1)$ & $28(15.6)$ & $39(18.4)$ & \multirow[t]{2}{*}{0.457} \\
\hline No & $325(82.9)$ & $152(84.4)$ & $173(81.6)$ & \\
\hline Fish intake (g/day) & $37.2 \pm 33.9$ & $37.0 \pm 30.1$ & $37.4 \pm 36.9$ & 0.924 \\
\hline \multicolumn{5}{|l|}{ AMC and/or ATG positive } \\
\hline Yes & $40(10.2)$ & $19(10.6)$ & $21(9.9)$ & \multirow[t]{2}{*}{0.832} \\
\hline No & $352(89.8)$ & $161(89.4)$ & $191(90.1)$ & \\
\hline \multicolumn{5}{|c|}{ Blood sampling period for PFOS and PFOA } \\
\hline Before delivery & $286(73.0)$ & $133(73.9)$ & $153(72.2)$ & \multirow[t]{2}{*}{0.703} \\
\hline After delivery & $106(27.0)$ & $47(26.1)$ & $59(27.8)$ & \\
\hline $\begin{array}{l}\text { Blood sampling period of TSH, FT4 } \\
\text { (weeks of pregnancy) }\end{array}$ & $11.1 \pm 2.6$ & $11.2 \pm 2.9$ & $11.1 \pm 2.3$ & 0.740 \\
\hline PFOS (ng/mL) ${ }^{\mathrm{b}}$ & $5.2(1.6-12.3)$ & $5.2(1.6-12.2)$ & $5.3(1.6-12.6)$ & 0.985 \\
\hline PFOA $(\mathrm{ng} / \mathrm{mL})^{\mathrm{b}}$ & $1.2(\mathrm{LOD}-3.4)$ & $1.3(\mathrm{LOD}-3.6)$ & $1.2(\mathrm{LOD}-3.4)$ & 0.834 \\
\hline $\mathrm{TSH}(\mu \mathrm{U} / \mathrm{mL})^{\mathrm{b}}$ & $1.0(\mathrm{LOD}-4.1)$ & $1.0(\mathrm{LOD}-4.3)$ & 1.0 (LOD-4.0) & 0.835 \\
\hline FT4 $(\mathrm{ng} / \mathrm{mL})^{\mathrm{b}}$ & $0.99(0.62-1.67)$ & $0.98(0.58-1.84)$ & $1.00(0.72-1.67)$ & 0.269 \\
\hline
\end{tabular}

ATC antimicrosome antibody, ATG antithyroglobulin antibody, BMI body mass index, FT4 free thyroxine, LOD limit of detection, PFOA perfluorooctanoate, PFOS perfluorooctane sulfonate, SD standard deviation, TSH thyroid stimulating hormone

${ }^{a}$ Comparing the differences between mothers of male infants and mothers of female infant. $p$ Values were calculated by the $t$ test and the chi square test

b Median (95\% confidence interval)

relation to various maternal characteristics are shown in Table 3. PFOS and PFOA levels were significantly higher among women who were primiparous and whose blood samples were obtained before delivery. Maternal age at delivery was negatively associated with PFOS levels among mothers of total infants and female infants. Negative correlation between maternal age at delivery and PFOA level was found only among mothers of female infants. As for mothers of male infants, PFOA level of mothers who smoked during pregnancy was significantly lower than that of non-smoking mothers, and inverse correlation was found between PFOA level and blood sampling period of TSH, FT4 (weeks of pregnancy), while those difference were not found among mothers of total and female infants.
The results of the correlations and the multiple linear regression analyses between maternal PFOS/PFOA concentrations and maternal TSH/FT4 are shown in Table 4. Maternal PFOS was significantly correlated with decreased maternal TSH levels. After adjustment for covariates, PFOS levels were inversely associated with TSH levels in mothers of total male and female infants. There was no significant association between PFOS and FT4. PFOA showed no significant association with either TSH or FT4.

Table 5 shows the results of the correlations and multiple linear regression analyses between maternal PFOS/ PFOA concentrations and infant TSH/FT4. There is no significant correlation between maternal PFCs and infants' THs. Among total infants, we observed a significantly positive association between maternal PFOS and infant 
Table 2 Comparisons of infants' characteristics and the concentrations of TSH and FT4 by infant sex

\begin{tabular}{llll}
\hline Characteristics of infants & $\begin{array}{l}\text { Total infants }(n=392) \\
\text { Median } \pm \text { SD or } n(\%)\end{array}$ & $\begin{array}{l}\text { Male infants }(n=180) \\
\text { Median } \pm \text { SD or } n(\%)\end{array}$ & $\begin{array}{l}\text { Female infants }(n=212) \\
\text { Median } \pm \text { SD or } n(\%)\end{array}$ \\
\hline $\begin{array}{l}\text { Gestational age at birth (weeks) } \\
\text { Birth weight }(\mathrm{g})\end{array}$ & $\begin{array}{l}38.9 \pm 1.5 \\
\text { Low birth weight }(<2500 \mathrm{~g})\end{array}$ & $38.9 \pm 1.3$ & $38.9 \pm 1.6$ \\
$\quad$ Yes & $25(6.4)$ & $3075.6 \pm 380.9$ & $3038.0 \pm 417.5$ \\
$\begin{array}{l}\text { Cesarean section } \\
\text { Yes }\end{array}$ & $10(5.6)$ & $15(7.1)$ \\
$\begin{array}{l}\text { Blood sampling (day after birth) } \\
\text { of TSH and FT4 }\end{array}$ & $4.5 \pm 0.9$ & $35(19.4)$ & $42(19.8)$ \\
$\begin{array}{l}\text { TSH }(\mu \mathrm{U} / \mathrm{mL})^{\mathrm{b}} \\
\text { FT4 }(\mathrm{ng} / \mathrm{mL})^{\mathrm{b}}\end{array}$ & $2.2($ LOD-7.9) & $4.5 \pm 1.0$ & $4.5 \pm 0.9$ \\
\hline
\end{tabular}

$A T C$ antimicrosome antibody, $A T G$ antithyroglobulin antibody, BMI body mass index, FT4 free thyroxine, LOD limit of detection, PFOA perfluorooctanoate, PFOS perfluorooctane sulfonate, SD standard deviation, TSH thyroid stimulating hormone

${ }^{\text {a }}$ Comparing the differences between mothers of male infants and mothers of female infant. $p$ Values were calculated by the $t$ test and the chi square test

b Median (95\% confidence interval)

TSH levels in the adjusted model. After stratifying by gender, we also observed a positive association of PFOS with TSH levels in both male and female infants. No significant association was found between PFOS and infant FT4. PFOA showed no significant association with infant TSH or FT4.

The relationships between quartile PFOS concentrations $(1.5 \leq$ quartile $1 \leq 3.5 \mathrm{ng} / \mathrm{mL}, 3.5<$ quartile $2 \leq 5.2 \mathrm{ng} /$ $\mathrm{mL}, \quad 5.2<$ quartile $\quad 3 \leq 7.0 \mathrm{ng} / \mathrm{mL}, \quad 7.0<$ quartile $4 \leq 16.2 \mathrm{ng} / \mathrm{mL}$ ) in maternal serum and TSH levels of mothers and infants are shown in Fig. 1. Maternal TSH showed a significantly decreasing trend in relation to quartile PFOS. Infant TSH showed an increasing trend in relation to the PFOS quartile in total infants although this was not significant after stratifying by sex.

\section{Discussion}

The fetal period is a particularly vulnerable stage during which many critical neurodevelopmental events occur. THs are known to be crucial for fetal growth [22]. Our results suggest that PFOS exposure at common environmental levels during pregnancy is likely to decrease maternal TSH and increase infant TSH. Such subclinical hyper- or hypothyroidism [23] might lead to the imbalance of FT4, an important hormone for fetal neurodevelopment.

PFOS and PFOA levels in this study were lower compared to other areas of Japan and Korea [24]. Moreover, mothers who had experienced delivery before the participation to this study had significantly lower levels of PFOS and PFOA, which is consistent with other study [25]. According to previous reports, PFOS and PFOA levels of pregnant women are lower than non-pregnant women, indicating the placental transfer to fetus [26, 27].

Three previous studies in Norway, Taiwan, and Canada have reported positive associations between serum PFOS levels in pregnant women and their TH levels [15-17]. Our data, which shows an inverse association between maternal PFOS concentration and maternal TSH, is inconsistent with these studies. There are some difficulties in comparisons due to the difference of the timing of blood collection for TH measurement between previous studies and ours. First, circulating $\mathrm{TH}$ levels during pregnancy greatly change according to the gestational week. In previous studies, maternal blood was obtained after the second trimester (between 15 and 18 gestational weeks for Webster et al. in Canada, in the second trimester and 3 days to 6 weeks post-delivery for Berg et al. in Norway, and in the third trimester for Wang et al. in Taiwan), while our study measured the levels during the first trimester (median 11.1 weeks of gestation). Fetal thyroid glands begin to secrete hormones after the second trimester of gestation $[28,29]$. During early pregnancy, fetuses rely on maternal THs, and the disruption of maternal TH homeostasis can affect both maternal and fetal health. Deficiency or imbalance of maternal THs likely disrupts normal neurological development of fetuses. Therefore, our study clarified the effect of maternal $\mathrm{THs}$ on fetuses in early gestational stages. Additionally, reproductive hormone levels also drastically change at each stage of pregnancy. The level of human chorionic gonadotropin (hCG) increases rapidly until 10 weeks of gestation, and decreases soon. Then, estrogen and progesterone gradually increase until delivery. One previous report showed that estrogen indirectly increased the thyroxine binding globulin [30], 
Table 3 Relationships between maternal factors and PFOS and PFOA concentrations in maternal serum

\begin{tabular}{|c|c|c|c|c|c|c|}
\hline \multirow[t]{2}{*}{ Characteristics of mothers } & \multicolumn{2}{|c|}{$\begin{array}{l}\text { Mothers of total infants } \\
(n=392)\end{array}$} & \multicolumn{2}{|c|}{$\begin{array}{l}\text { Mothers of male infants } \\
(n=180)\end{array}$} & \multicolumn{2}{|c|}{$\begin{array}{l}\text { Mothers of female infants } \\
(n=212)\end{array}$} \\
\hline & PFOS & PFOA & PFOS & PFOA & PFOS & PFOA \\
\hline Age (years) at delivery ${ }^{\mathrm{a}}$ & $r=-0.170^{* *}$ & $r=-0.071$ & $r=-0.115$ & $r=0.026$ & $r=-0.209 * *$ & $r=-0.152^{*}$ \\
\hline BMI $\left(\mathrm{kg} / \mathrm{m}^{2}\right)$ before pregnancy ${ }^{\mathrm{a}}$ & $r=-0.065$ & $r=-0.067$ & $r=-0.0046$ & $r=0.022$ & $r=-0.083$ & $r=-0.146^{*}$ \\
\hline \multicolumn{7}{|l|}{ Parity (times) ${ }^{\mathrm{b}, \mathrm{c}}$} \\
\hline 0 & $\begin{array}{l}6.0 \\
(1.8-13.4)^{* *}\end{array}$ & $\begin{array}{l}1.5 \\
(0.4-4.3)^{* *}\end{array}$ & $\begin{array}{l}6.1 \\
\quad(1.8-13.6)^{* *}\end{array}$ & $\begin{array}{l}1.5 \\
(0.6-4.7)^{* *}\end{array}$ & $\begin{array}{l}5.7 \\
\quad(1.6-13.9)^{* * *}\end{array}$ & $\begin{array}{l}1.5 \\
(0.3-4.2)^{* *}\end{array}$ \\
\hline$\geq 1$ & $4.8(1.5-11.1)$ & $0.9(0.3-2.7)$ & $4.9(1.4-10.2)$ & $0.9(0.3-2.6)$ & $4.7(1.6-11.3)$ & $0.9(0.3-2.9)$ \\
\hline \multicolumn{7}{|l|}{ Educational level (years) ${ }^{\mathrm{b}}$} \\
\hline$\leq 12$ & $5.0(1.7-12.2)$ & $1.2(0.3-3.7)$ & $4.9(1.4-12.1)$ & $1.2(0.3-4.2)$ & $5.1(1.7-12.9)$ & $1.2(0.3-3.7)$ \\
\hline$\geq 13$ & $5.5(1.6-12.7)$ & $1.3(0.3-3.4)$ & $6.1(1.6-13.3)$ & $1.3(0.3-3.7)$ & $5.4(1.5-12.7)$ & $1.3(0.3-2.8)$ \\
\hline \multicolumn{7}{|l|}{ Annual income (millions of yens) ${ }^{b}$} \\
\hline$<5$ & $5.2(1.6-12.3)$ & $1.2(0.3-3.7)$ & $5.2(1.5-12.4)$ & $1.2(0.3-4.3)$ & $5.1(1.6-12.4)$ & $1.2(0.3-3.6)$ \\
\hline$\geq 5$ & $5.5(1.6-13.1)$ & $1.3(0.3-2.6)$ & $5.6(2.0-13.0)$ & $1.3(0.3-2.6)$ & $5.5(1.6-13.2)$ & $1.3(0.3-3.0)$ \\
\hline \multicolumn{7}{|l|}{ Smoking during pregnancy ${ }^{\mathrm{b}}$} \\
\hline Yes & $4.8(1.5-12.5)$ & $1.1(0.3-3.1)$ & $4.5(1.5-12.1)$ & $\begin{array}{l}1.0 \\
(0.3-2.4)^{*}\end{array}$ & $5.2(1.5-13.3)$ & $1.1(0.3-3.2)$ \\
\hline No & $5.3(1.6-12.4)$ & $1.3(0.3-3.6)$ & $5.4(1.6-12.5)$ & $1.3(0.3-3.8)$ & $5.3(1.6-12.6)$ & $1.3(0.3-3.5)$ \\
\hline Fish intake $(\mathrm{g} / \text { day })^{\mathrm{a}}$ & $r=0.000$ & $r=-0.052$ & $r=-0.006$ & $r=-0.026$ & $r=0.028$ & $r=-0.078$ \\
\hline \multicolumn{7}{|l|}{ AMC and/or ATG positive ${ }^{\mathrm{b}}$} \\
\hline Yes & $5.8(1.4-13.3)$ & $1.4(0.3-3.8)$ & $4.2(1.4-12.1)$ & $1.5(0.6-2.4)$ & $5.8(2.5-13.3)$ & $1.1(0.3-3.8)$ \\
\hline No & $5.2(1.6-12.3)$ & $1.2(0.3-3.4)$ & $5.2(1.8-12.4)$ & $1.2(0.3-3.7)$ & $5.2(1.6-12.4)$ & $1.2(0.3-3.3)$ \\
\hline \multicolumn{7}{|l|}{ Blood sampling period for PFOS and $\mathrm{PFOA}^{\mathrm{b}}$} \\
\hline Before delivery & $\begin{array}{l}5.7 \\
\quad(1.8-12.6)^{* *}\end{array}$ & $\begin{array}{l}1.3 \\
(0.3-3.7)^{* *}\end{array}$ & $\begin{array}{l}5.6 \\
(2.0-12.6)^{* *}\end{array}$ & $\begin{array}{l}1.4 \\
(0.3-4.1)^{*}\end{array}$ & $\begin{array}{l}5.8 \\
\quad(1.7-12.8)^{* * *}\end{array}$ & $\begin{array}{l}1.3 \\
(0.3-3.5)^{* *}\end{array}$ \\
\hline After delivery & $3.8(1.4-11.2)$ & $1.0(0.3-2.5)$ & $4.0(1.4-10.4)$ & $1.0(0.3-2.6)$ & $3.4(1.4-13.1)$ & $1.0(0.3-2.6)$ \\
\hline $\begin{array}{l}\text { Blood sampling period of TSH, FT4 (weeks } \\
\text { of pregnancy) }^{\mathrm{a}}\end{array}$ & $r=0.023$ & $r=-0.077$ & $r=-0.016$ & $r=-0.152^{*}$ & $r=0.053$ & $r=-0.011$ \\
\hline
\end{tabular}

ATC antimicrosome antibody, $A T G$ antithyroglobulin antibody, BMI body mass index, FT4 free thyroxine, $L O D$ limit of detection, $P F O A$ perfluorooctanoate, $P F O S$ perfluorooctane sulfonate, $T S H$ thyroid stimulating hormone

$p$ Values were calculated by the Mann-Whitney $U$ test. $* p<0.05, * * p<0.01$

${ }^{\mathrm{a}} p$ Values were calculated by the Spearman correlation test

b Median (95\% confidence interval) (ng/mL)

which suggests the hormonal interaction. Therefore, the differences of the blood sampling timing may lead the inconsistent results between ours and previous studies due to the different hormonal balances. Second, the concentrations of PFOS in serum were different in previous studies compared to ours. Two of the previous studies reported higher PFOS concentrations in serum (Berg et al. [16], $8.03 \mathrm{ng} / \mathrm{mL}$; Wang et al. [17], $12.73 \mathrm{ng} / \mathrm{mL}$ ) compared to our study $(5.3 \mathrm{ng} / \mathrm{mL})$, while Webster et al. [15] reported a lower concentration $(4.8 \mathrm{ng} / \mathrm{mL})$. Although whether PFOS and PFOA are metabolized in humans is still unclear, our results suggest an association between human thyroid homeostasis and exposure to background environmental levels of PFOS.

There are two previous studies regarding the effect of PFCs on infant THs. Kim et al. [18] investigated the association of PFCs in maternal blood sera and cord blood with infant TH in cord blood among participants in South Korea $(n=44)$. They reported negative correlation between maternal PFOS and fetal total T3, and between maternal perfluorotridecanoic acid (PFTrDA) and total T3 and T4 in fetuses; moreover, maternal PFOA positively correlated with fetal TSH. A Dutch study reported that a high level of PFOA in cord blood was associated with increased total T4 levels in infant girls as measured during heel prick blood draws $(n=83)$ [19]. We found a significant positive association between maternal PFOS levels and infants' TSH in a larger sample size than those of previous studies. Our result is partially in line with Kim et al. that PFOS in maternal serum was associated with increased TSH, though their result was not significant. There are some discrepancies between our results and 
Table 4 Spearman's correlation test and multiple linear regression analyses of maternal TSH and FT4 by maternal PFOS and PFOA levels

\begin{tabular}{|c|c|c|c|c|c|c|c|c|c|c|c|c|}
\hline & \multicolumn{6}{|l|}{ PFOS } & \multicolumn{6}{|l|}{ PFOA } \\
\hline & \multicolumn{2}{|c|}{ Correlation $^{\mathrm{a}}$} & \multicolumn{2}{|l|}{ Crude $^{\mathrm{b}}$} & \multicolumn{2}{|c|}{ Adjusted $^{\mathrm{b}, \mathrm{c}}$} & \multicolumn{2}{|c|}{ Correlation $^{\mathrm{a}}$} & \multicolumn{2}{|c|}{ Crude $^{\mathrm{b}}$} & \multicolumn{2}{|c|}{ Adjusted $^{\mathrm{b}, \mathrm{c}}$} \\
\hline & $\rho$ & $p$ value & $\beta$ & $p$ value & $\beta$ & $p$ value & $\rho$ & $p$ value & $\beta$ & $p$ value & $\beta$ & $p$ value \\
\hline \multicolumn{13}{|c|}{ All mothers $(n=392)$} \\
\hline TSH & -0.179 & $<0.001$ & -0.187 & $<0.001$ & -0.214 & $<0.001$ & 0.042 & 0.412 & 0.056 & 0.272 & 0.039 & 0.478 \\
\hline FT4 & 0.083 & 0.100 & 0.069 & 0.176 & 0.061 & 0.236 & 0.023 & 0.651 & 0.023 & 0.646 & 0.004 & 0.946 \\
\hline \multicolumn{13}{|c|}{ Mothers of male infants $(n=180)$} \\
\hline TSH & -0.194 & 0.009 & -0.219 & 0.003 & -0.246 & 0.002 & -0.034 & 0.652 & 0.004 & 0.959 & -0.067 & 0.435 \\
\hline FT4 & 0.118 & 0.114 & 0.113 & 0.130 & 0.093 & 0.208 & 0.058 & 0.437 & 0.023 & 0.755 & 0.023 & 0.762 \\
\hline \multicolumn{13}{|c|}{ Mothers of female infants $(n=212)$} \\
\hline TSH & -0.164 & 0.017 & -0.163 & 0.018 & -0.210 & 0.005 & 0.108 & 0.119 & 0.101 & 0.142 & 0.114 & 0.124 \\
\hline FT4 & 0.056 & 0.422 & 0.032 & 0.648 & 0.030 & 0.684 & -0.017 & 0.810 & 0.024 & 0.727 & 0.006 & 0.936 \\
\hline
\end{tabular}

$\beta$ with $p<0.05$ are shown in bold

FT4 free thyroxine, PFOA perfluorooctanoate, PFOS perfluorooctane sulfonate, TSH thyroid stimulating hormone

a Spearman's correlation test

b Concentrations of PFOS, PFOA, TSH, and FT4 are log 10 transformed

c Adjusted for maternal age at delivery, BMI, parity, educational level, thyroid antibody, intake of seaweed, blood sampling period before/after delivery for PFOS and PFOA, and gestational week at which blood sampling was obtained for TSH and FT4

Table 5 Spearman's correlation test and multiple linear regression analyses of infant TSH and FT4 by maternal PFOS and PFOA levels

\begin{tabular}{|c|c|c|c|c|c|c|c|c|c|c|c|c|}
\hline & \multicolumn{6}{|l|}{ PFOS } & \multicolumn{6}{|l|}{ PFOA } \\
\hline & \multicolumn{2}{|c|}{ Correlation $^{\mathrm{a}}$} & \multicolumn{2}{|l|}{ Crude $^{\mathrm{b}}$} & \multicolumn{2}{|c|}{ Adjusted $^{\mathrm{b}, \mathrm{c}}$} & \multicolumn{2}{|c|}{ Correlation $^{\mathrm{a}}$} & \multicolumn{2}{|l|}{ Crude $^{\mathrm{b}}$} & \multicolumn{2}{|c|}{ Adjusted $^{\mathrm{b}, \mathrm{c}}$} \\
\hline & $\rho$ & $p$ value & $\beta$ & $p$ value & $\beta$ & $p$ value & $\rho$ & $p$ value & $\beta$ & $p$ value & $\beta$ & $p$ value \\
\hline \multicolumn{13}{|c|}{ All infants $(n=392)$} \\
\hline TSH & 0.061 & 0.229 & 0.080 & 0.112 & 0.177 & 0.001 & -0.077 & 0.127 & -0.066 & 0.190 & -0.014 & 0.801 \\
\hline FT4 & 0.015 & 0.774 & 0.003 & 0.961 & -0.043 & 0.452 & -0.012 & 0.815 & 0.014 & 0.786 & 0.003 & 0.960 \\
\hline \multicolumn{13}{|c|}{ Male infants $(n=180)$} \\
\hline TSH & 0.057 & 0.444 & 0.084 & 0.264 & 0.205 & 0.014 & -0.146 & 0.051 & -0.125 & 0.095 & -0.095 & 0.277 \\
\hline FT4 & 0.012 & 0.877 & -0.040 & 0.598 & -0.037 & 0.670 & -0.031 & 0.678 & 0.033 & 0.658 & 0.023 & 0.795 \\
\hline \multicolumn{13}{|c|}{ Female infants $(n=212)$} \\
\hline TSH & 0.071 & 0.301 & 0.081 & 0.241 & 0.173 & 0.021 & -0.017 & 0.808 & -0.013 & 0.847 & 0.054 & 0.465 \\
\hline FT4 & 0.022 & 0.753 & 0.032 & 0.642 & -0.044 & 0.567 & 0.001 & 0.988 & -0.001 & 0.990 & -0.017 & 0.819 \\
\hline
\end{tabular}

$\beta$ with $p<0.05$ are shown in bold

FT4 free thyroxine, PFOA perfluorooctanoate, PFOS perfluorooctane sulfonate, TSH thyroid stimulating hormone

a Spearman's correlation test

b Concentrations of PFOS, PFOA, TSH, and FT4 are log 10 transformed

c Adjusted for maternal factors (maternal age at delivery, parity, thyroid antibody, blood sampling period before/after delivery for PFOS and PFOA, and values of TSH and FT4); and infant factors (gestational weeks at birth, low birth weight $<2500 \mathrm{~g}$, and mode of delivery)

previous studies. We measured free T4 levels, which we assumed would be a more reliable indicator of hyper- or hypothyroidism, because FT4 is not affected by the concentration of binding proteins, while two previous studies used the data of total T4. Our results of elevated TSH without FT4 change indicate that prenatal PFOS exposure may cause subclinical hypothyroidism in fetuses. In addition, we added maternal TH levels as the confounders in the multiple regression analysis, considering the maternal TH effects on infants' TH. The correlations between maternal TSH and infants' FT4 were found to be significant inversely (Spearman's $\rho=-0.104, p=0.040$ ) in this study, which is consistent with one previous report that showed the inverse relationship between maternal TSH and neonatal total T4 [31]. Although maternal TSH is not transferred to fetus, the associations of PFOS levels with 

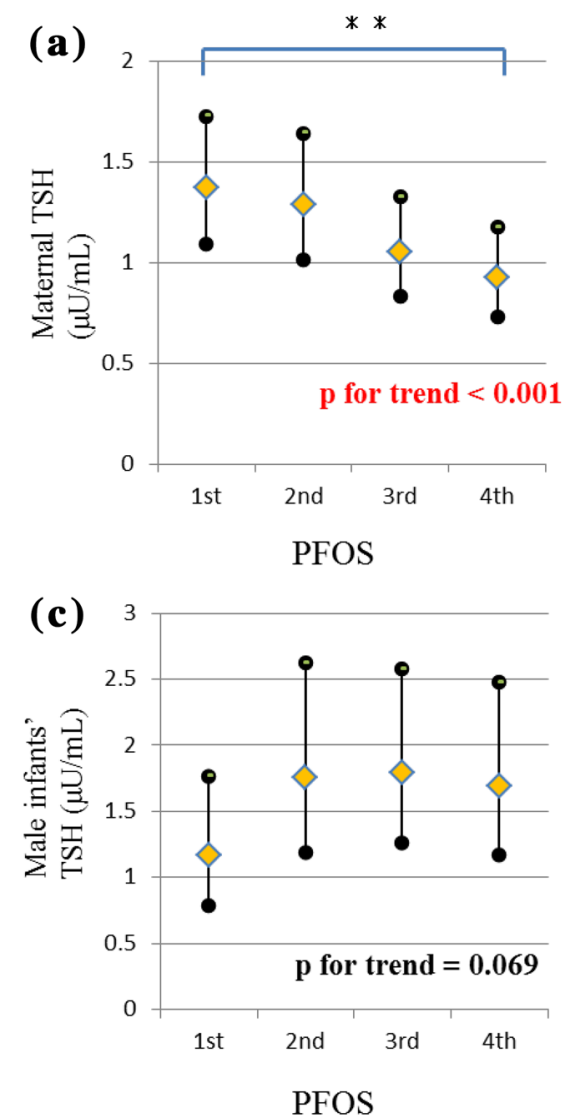

Fig. 1 Least square means of maternal and infant TSH, according to maternal serum PFOS concentration quartiles $(1.5 \leq$ quartile $1 \leq 3.5 \mathrm{ng} / \mathrm{mL}, \quad 3.5<$ quartile $\quad 2 \leq 5.2 \mathrm{ng} / \mathrm{mL}, \quad 5.2<$ quartile $3 \leq 7.0 \mathrm{ng} / \mathrm{mL}, 7.0<$ quartile $4 \leq 16.2 \mathrm{ng} / \mathrm{mL}$ ). a Maternal TSH according to PFOS, $\mathbf{b}$ total infant TSH according to PFOS, $\mathbf{c}$ male infant TSH according to PFOS, $\mathbf{d}$ female infant TSH according to PFOS. Results are based on multiple linear regression models that were adjusted for the following factors: for a maternal age at delivery (years), BMI before pregnancy $\left(\mathrm{kg} / \mathrm{m}^{2}\right)$, parity $(0 / \geq 1)$, educational level ( $\leq 12 / \geq 13$ years), positivity for ATG and/or AMC (yes/no), intake of seaweed more than once a week (yes/no), blood sampling period for PFOS and PFOA (before/after delivery), and gestational

decreased TSH among mothers might disrupt infants' hormone levels. Further study is needed to clarify the association between disrupted TH by PFOS exposure in maternal serum and infants' TH levels. It is noted that our previous study reported the inverse association between maternal PFOS and birth weight among female infants [11]. In this study, there was a significant inverse correlation between birth weight and TSH $(r=-0.155)$. Decreased birth weight by PFOS exposure might lead to affect TSH level of infants, however, our results showed the increased TSH by PFOS exposure. Further study is needed to investigate the intermediation of known relationships by PFC exposure. Since the quartile analysis showed the significant $p$ for trend $(p$ for trend $<0.05$ ) in total infants and the tendency ( $p$ for trend $<0.10$ ) in female
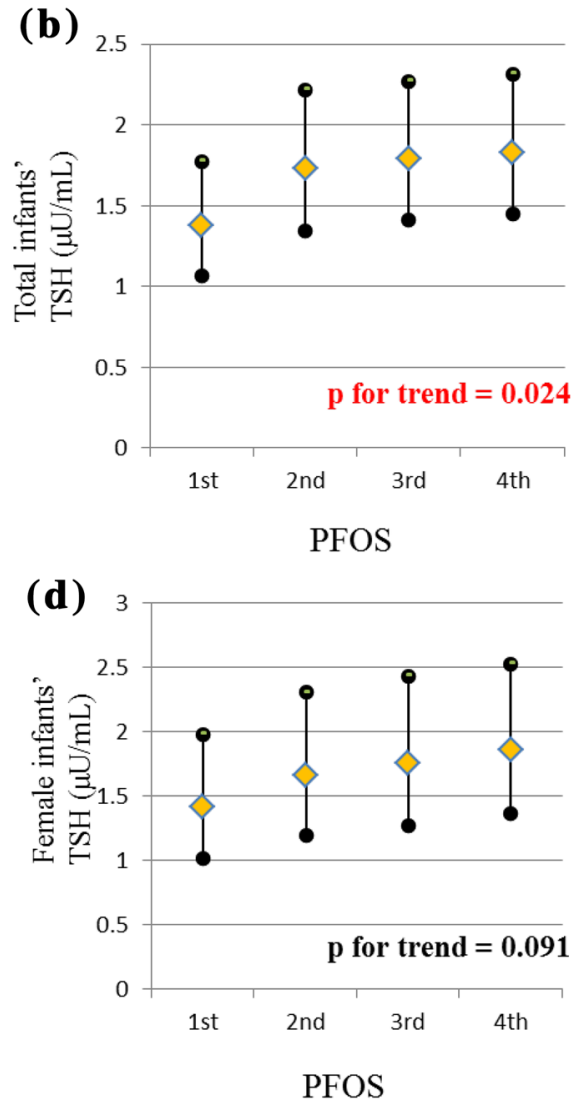

week of blood sampling for TSH and FT4 (continuous variable); and for b-d maternal factors [maternal age at delivery (years), parity ( $0 /$ $\geq 1$ ), positivity for ATG and/or AMC (yes/no), blood sampling period for PFOS and PFOA (before/after delivery), log 10 values of TSH and FT4 (continuous variable)] and infant factors [gestational weeks at birth (continuous variable), low birth weight (yes/no), and born via cesarean section (yes/no)]. $* * p<0.01$ compared to the first quartile, as calculated using the Hsu-Dunnett's method. LSMs are indicated in rhombus and the black dots depict the upper and lower $95 \%$ CI. ATC antimicrosome antibody, $A T G$ antithyroglobulin antibody, $B M I$ body mass index, FT4 free thyroxine, PFOA perfluorooctanoate, PFOS perfluorooctane sulfonate, TSH thyroid stimulating hormone

infants for the increasing TSH, our results indicate the PFOS dose-response toxicity for TSH even at low exposure level. On the contrary, male infants' TSH showed highest levels at 2nd quartiles of PFOS and slightly decreased at $3 \mathrm{rd}$ and 4 th quartiles. That suggests no doseresponse relationships among male infants. Although the reason for sex differences is not understood, one explanation for the sex difference is the faster elimination time of PFCs in female compared to male in animal studies [32, 33].

We found that the PFOA level had no effect on TSH or FT4, implying that PFOS is more influential than PFOA on human THs. One study observed the negative association of PFOA levels with free T4 and positive association with T3 among male adults exposed to PFOA occupationally 
[34]. Additionally, two studies regarding the effects of in utero exposure on infants' THs reported the significant associations of PFOA with TSH or FT4 [18, 19]. Our results were inconsistent with those previous studies. Concentrations of PFOA in cord blood were reported to be much closer to concentrations in maternal blood compared to PFOS [8, 35]. This suggests that PFOA transfers more easily across the placental barrier than PFOS. However, Olsen et al. [6] reported that the half-life of elimination of PFOS was longer than that of PFOA. One explanation for our results is that PFOS may have stronger effects than PFOA on THs in this study by remaining in maternal serum at a higher concentration due to its longer elimination time.

There are some inconsistencies between human and animal studies, such as higher doses of PFCs used in animal studies as well as physiologic differences. In rats and mice, PFOS has been reported to reduce concentrations of T3 and T4 without affecting stimulation of TSH $[13,14]$. Although our results differed in that PFOS had no effect on FT4 in either maternal or infant serum, findings in rodents are difficult to extrapolate to humans because the high level of exposure to PFOS and PFOA in rodents is probably not comparable to the chronic low level exposures examined in our study. Moreover, PFOS may remain in the human body much longer than in other animals. A serum elimination half-life of 7.5 days has been reported for rats [36], whereas the half-life is 200 days in monkeys [37] and approximately 5.4 years in humans [6]. In animal experiments, repeated doses maintain the high serum levels even with fast elimination, while in human serum levels also remain low but stable due to long half-lives. Another discrepancy is the role of transthyretin (TTR). Although PFOS has been reported to competitively bind to TTR [38], only $10 \%$ of circulating T4 binds to TTR in humans, while the percentage in rats is $75 \%$.

A major strength of this study is its prospective design, which allowed us to estimate the effects of prenatal PFC exposure on fetal thyroid functions using prenatal and perinatal blood samples. However, there are several limitations in this study. The detection of PFOS and PFOA was also limited by the amount of blood obtained from mothers. Compared to the excluded women in original cohort population (122 mothers), the median levels of maternal PFOS and PFOA did not significantly differ between the analyzed participants (PFOS $5.2 \mathrm{ng} / \mathrm{mL}$; PFOA $1.2 \mathrm{ng} / \mathrm{mL}$ ) and the excluded participants (PFOS $5.0 \mathrm{ng} / \mathrm{mL}$, PFOA $1.5 \mathrm{ng} /$ $\mathrm{mL})$. However, the infants who were included in our analyses had a higher gestational age and a heavier birth weight (data not shown), which indicates a possible selection bias. Among the 392 mother-infant pairs, blood samples were obtained from 106 mothers at a later time post-delivery due to anemia, and these post-delivery blood samples exhibited significantly lower PFOS and PFOA (before delivery: $\mathrm{PFOS}=5.2$ and $\mathrm{PFOA}=1.4 \mathrm{ng} / \mathrm{mL}$; post-delivery: $\mathrm{PFOS}=3.5 \mathrm{ng} / \mathrm{mL}$ and $\mathrm{PFOA}=1.3 \mathrm{ng} /$ $\mathrm{mL})$. Although there is no report regarding PFOS and PFOA half-lives among pregnant women, PFOS and PFOA were reported to have long half-lives in adult human serum (5.4 and 3.8 years, respectively). We believed that the postdelivery samples accurately reflected these women's PFC levels during their pregnancy. In addition, PFC concentrations during pregnancy are reported to decrease across trimesters [26]. We found the significant inverse correlation between PFOS and PFOA levels and gestational weeks of blood sampling for the measurement of PFOS and PFOA during pregnancy (PFOS; $r=-0.224$, PFOA; $r=-0.177)$. We also analyzed using continuous variables of gestational weeks for PFOS and PFOA measurement, and the significance and direction of the results were almost same as dichotomous variables. Therefore, we retained those mothers whose blood samples were obtained after delivery to increase this study's statistical power, and also included the blood sampling period as a potential confounder in the adjusted models. It is noted that we also included 40 mothers with AMC and/or ATG positive in this study. They were not under medical treatments at the recruitment, however, TSH levels of 40 mothers with AMC and/or ATG positive (median $1.5 \mu \mathrm{U} / \mathrm{mL}$ ) were significantly higher than those of 352 mothers with AMC and/or ATG negative (median $0.9 \mu \mathrm{U} / \mathrm{mL}$ ), and FT4 levels of 40 mothers with AMC and/or ATG positive (median $0.91 \mathrm{ng} /$ $\mathrm{mL}$ ) were significantly lower than those of 352 mothers with AMC and/or ATG negative (median $1.00 \mathrm{ng} / \mathrm{mL}$ ), respectively. We did the analysis excluding 40 women with AMC/ATC positive, and the results were almost same as Tables 4 and 5 in the significance and directions. Thus, we included these 40 women in this study, adding the presence of AMC and/or ATG into one of confounders. Finally, we did not have data for hCG levels. TSH levels in early pregnancy stages are suppressed because of the elevation of hCG [39, 40]. Maternal TH levels were measured at 11.1 weeks of gestation in our study, when hCG is usually very high. However, all $\mathrm{TH}$ data were obtained before the 20th weeks of gestation to ensure that there was an actual relationship between PFOS and TSH.

In conclusion, our study revealed an inverse association between maternal PFOS concentration and maternal TSH during early gestation, and a positive association between maternal PFOS concentration and infant TSH at background-level PFOS exposures. Our group previously reported a decreasing trend for PFOS and PFOA levels, and an increasing trend for PFC levels with longer carbon chains (e.g., perfluorononanoic acid and perfluorodecanoic acid), in the plasma of pregnant women between 2003 and 2011 [41]. Therefore, future studies are required to verify the effects of PFCs with longer carbon chains on fetal 
thyroid functions. Moreover, multi-exposure analysis is required because our study reported that most concentrations of individual chemicals, such as polychlorinated biphenyls, PFOS, PFOA and mercury, were correlated due to the similar exposure sources [42]. Additionally, these studies should also investigate the long-term effect of altered hormone levels on fetal neurodevelopment.

Acknowledgments We thank the staff at Sapporo Toho Hospital and the participating mothers and infants for their collaboration. We also thank N. Koibuchi for instruction on thyroid homeostasis function, and R. Ito for the measurement of PFOS and PFOA. This study was supported by Grants-in-Aid for Health Scientific Research from the Japanese Ministry of Health, Labour and Welfare (20805801); and by Grants-in-Aid for Scientific Research from the Japanese Ministry of Education, Culture, Sports, Science, and Technology (13307015).

\section{Compliance with ethical standards}

Conflict of interest The authors declare that they have no conflicts of interest.

Informed consent This study was conducted with written informed consent from all subjects and was approved by the ethics committees of Hokkaido University Graduate School of Medicine.

\section{References}

1. Fromme H, Tittlemier SA, Volkel W, Wilhelm M, Twardella D. Perfluorinated compounds-exposure assessment for the general population in Western countries. Int J Hyg Environ Health. 2009;212(3):239-70.

2. Butenhoff JL, Olsen GW, Pfahles-Hutchens A. The applicability of biomonitoring data for perfluorooctanesulfonate to the environmental public health continuum. Environ Health Perspect. 2006;114(11):1776-82.

3. Calafat AM, Kuklenyik Z, Reidy JA, Caudill SP, Tully JS, Needham LL. Serum concentrations of 11 polyfluoroalkyl compounds in the US population: data from the national health and nutrition examination survey (NHANES). Environ Sci Technol. 2007;41(7):2237-42.

4. Harada K, Saito N, Inoue K, Yoshinaga T, Watanabe T, Sasaki S, Kamiyama S, Koizumi A. The influence of time, sex and geographic factors on levels of perfluorooctane sulfonate and perfluorooctanoate in human serum over the last 25 years. J Occup Health. 2004;46(2):141-7.

5. Kannan K, Corsolini S, Falandysz J, Fillmann G, Kumar KS, Loganathan BG, Mohd MA, Olivero J, Van Wouwe N, Yang JH, et al. Perfluorooctanesulfonate and related fluorochemicals in human blood from several countries. Environ Sci Technol. 2004;38(17):4489-95.

6. Olsen GW, Burris JM, Ehresman DJ, Froehlich JW, Seacat AM, Butenhoff JL, Zobel LR. Half-life of serum elimination of perfluorooctanesulfonate, perfluorohexanesulfonate, and perfluorooctanoate in retired fluorochemical production workers. Environ Health Perspect. 2007;115(9):1298-305.

7. Inoue K, Okada F, Ito R, Kato S, Sasaki S, Nakajima S, Uno A, Saijo Y, Sata F, Yoshimura Y, et al. Perfluorooctane sulfonate (PFOS) and related perfluorinated compounds in human maternal and cord blood samples: assessment of PFOS exposure in a susceptible population during pregnancy. Environ Health Perspect. 2004;112(11):1204-7.

8. Midasch O, Drexler H, Hart N, Beckmann MW, Angerer J. Transplacental exposure of neonates to perfluorooctanesulfonate and perfluorooctanoate: a pilot study. Int Arch Occup Environ Health. 2007;80(7):643-8.

9. Apelberg BJ, Witter FR, Herbstman JB, Calafat AM, Halden RU, Needham LL, Goldman LR. Cord serum concentrations of perfluorooctane sulfonate (PFOS) and perfluorooctanoate (PFOA) in relation to weight and size at birth. Environ Health Perspect. 2007;115(11):1670-6.

10. Chen MH, Ha EH, Liao HF, Jeng SF, Su YN, Wen TW, Lien GW, Chen CY, Hsieh WS, Chen PC. Perfluorinated compound levels in cord blood and neurodevelopment at 2 years of age. Epidemiology (Cambridge, Mass). 2013;24(6):800-8.

11. Washino N, Saijo Y, Sasaki S, Kato S, Ban S, Konishi K, Ito R, Nakata A, Iwasaki Y, Saito K, et al. Correlations between prenatal exposure to perfluorinated chemicals and reduced fetal growth. Environ Health Perspect. 2009;117(4):660-7.

12. Kishi R, Nakajima T, Goudarzi H, Kobayashi S, Sasaki S, Okada E, Miyashita C, Itoh S, Araki A, Ikeno T, et al. The association of prenatal exposure to perfluorinated chemicals with maternal essential and long-chain polyunsaturated fatty acids during pregnancy and the birth weight of their offspring: the Hokkaido Study. Environ Health Perspect. 2015;123:1038-45.

13. Luebker DJ, York RG, Hansen KJ, Moore JA, Butenhoff JL. Neonatal mortality from in utero exposure to perfluorooctanesulfonate (PFOS) in Sprague-Dawley rats: dose-response, and biochemical and pharmacokinetic parameters. Toxicology. 2005;215(1-2):149-69.

14. Lau C, Thibodeaux JR, Hanson RG, Rogers JM, Grey BE, Stanton ME, Butenhoff JL, Stevenson LA. Exposure to perfluorooctane sulfonate during pregnancy in rat and mouse. II: postnatal evaluation. Toxicol Sci. 2003;74(2):382-92.

15. Webster GM, Venners SA, Mattman A, Martin JW. Associations between perfluoroalkyl acids (PFASs) and maternal thyroid hormones in early pregnancy: a population-based cohort study. Environ Res. 2014;133:338-47.

16. Berg V, Nost TH, Hansen S, Elverland A, Veyhe AS, Jorde R, Odland JO, Sandanger TM. Assessing the relationship between perfluoroalkyl substances, thyroid hormones and binding proteins in pregnant women; a longitudinal mixed effects approach. Environ Int. 2015;77:63-9.

17. Wang Y, Rogan WJ, Chen PC, Lien GW, Chen HY, Tseng YC, Longnecker MP, Wang SL. Association between maternal serum perfluoroalkyl substances during pregnancy and maternal and cord thyroid hormones: Taiwan maternal and infant cohort study. Environ Health Perspect. 2014;122(5):529-34.

18. Kim S, Choi K, Ji K, Seo J, Kho Y, Park J, Kim S, Park S, Hwang I, Jeon J, et al. Trans-placental transfer of thirteen perfluorinated compounds and relations with fetal thyroid hormones. Environ Sci Technol. 2011;45(17):7465-72.

19. de Cock M, de Boer MR, Lamoree M, Legler J, van de Bor M. Prenatal exposure to endocrine disrupting chemicals in relation to thyroid hormone levels in infants-a Dutch prospective cohort study. Environ Health. 2014;13:106.

20. Kishi R, Kobayashi S, Ikeno T, Araki A, Miyashita C, Itoh S, Sasaki S, Okada E, Kobayashi S, Kashino I, et al. Ten years of progress in the Hokkaido birth cohort study on environment and children's health: cohort profile-updated 2013. Environ Health Prev Med. 2013;18(6):429-50.

21. Okada E, Sasaki S, Saijo Y, Washino N, Miyashita C, Kobayashi S, Konishi K, Ito YM, Ito R, Nakata A, et al. Prenatal exposure to perfluorinated chemicals and relationship with allergies and infectious diseases in infants. Environ Res. 2012;112:118-25. 
22. Williams FL, Mires GJ, Barnett C, Ogston SA, van Toor H, Visser TJ, Hume R. Transient hypothyroxinemia in preterm infants: the role of cord sera thyroid hormone levels adjusted for prenatal and intrapartum factors. J Clin Endocrinol Metab. 2005;90(8):4599-606.

23. Mortimer RH. Thyroid function tests. Aust Prescr. 2011;34(1):12-5.

24. Harada KH, Yang HR, Moon CS, Hung NN, Hitomi T, Inoue K, Niisoe T, Watanabe T, Kamiyama S, Takenaka K, et al. Levels of perfluorooctane sulfonate and perfluorooctanoic acid in female serum samples from Japan in 2008, Korea in 1994-2008 and Vietnam in 2007-2008. Chemosphere. 2010;79(3):314-9.

25. Papadopoulou E, Haug LS, Sabaredzovic A, Eggesbo M, Longnecker MP. Reliability of perfluoroalkyl substances in plasma of 100 women in two consecutive pregnancies. Environ Res. 2015;140:421-9.

26. Jain RB. Effect of pregnancy on the levels of selected perfluoroalkyl compounds for females aged 17-39 years: data from National Health and Nutrition Examination Survey 2003-2008. J Toxicol Environ Health Part A. 2013;76(7):409-21.

27. Javins B, Hobbs G, Ducatman AM, Pilkerton C, Tacker D, Knox SS. Circulating maternal perfluoroalkyl substances during pregnancy in the C8 Health Study. Environ Sci Technol. 2013;47(3):1606-13.

28. de Escobar GM, Obregon MJ, del Rey FE. Maternal thyroid hormones early in pregnancy and fetal brain development. Best Pract Res Clin Endocrinol Metab. 2004;18(2):225-48.

29. Obregon MJ, Calvo RM, Del Rey FE, de Escobar GM. Ontogenesis of thyroid function and interactions with maternal function. Endocr Dev. 2007;10:86-98.

30. Ben-Rafael Z, Struass JF 3rd, Arendash-Durand B, Mastroianni L $\mathrm{Jr}$, Flickinger GL. Changes in thyroid function tests and sex hormone binding globulin associated with treatment by gonadotropin. Fertil Steril. 1987;48(2):318-20.

31. Kuppens SM, Kooistra L, Wijnen HA, Vader HL, Hasaart TH, Oei SG, Vulsma T, Pop VJ. Neonatal thyroid screening results are related to gestational maternal thyroid function. Clin Endocrinol. 2011;75(3):382-7.

32. Lau C, Anitole K, Hodes C, Lai D, Pfahles-Hutchens A, Seed J. Perfluoroalkyl acids: a review of monitoring and toxicological findings. Toxicol Sci. 2007;99(2):366-94.
33. Zhang T, Sun H, Qin X, Gan Z, Kannan K. PFOS and PFOA in paired urine and blood from general adults and pregnant women: assessment of urinary elimination. Environ Sci Pollut Res Int. 2015;22(7):5572-9.

34. Olsen GW, Zobel LR. Assessment of lipid, hepatic, and thyroid parameters with serum perfluorooctanoate (PFOA) concentrations in fluorochemical production workers. Int Arch Occup Environ Health. 2007;81(2):231-46.

35. Fei C, McLaughlin JK, Tarone RE, Olsen J. Perfluorinated chemicals and fetal growth: a study within the Danish National Birth Cohort. Environ Health Perspect. 2007;115(11):1677-82.

36. Johnson JD, Gibson SJ, Ober RF. Absorption of FC-95-14C in rats after a single oral dose. St Paul: Riker Laboratories, Inc; 1979 (US EPA Docket No 8(e)HQ-1180-00374).

37. Seacat AM, Thomford PJ, Hansen KJ, Olsen GW, Case MT, Butenhoff JL. Subchronic toxicity studies on perfluorooctanesulfonate potassium salt in cynomolgus monkeys. Toxicol Sci. 2002;68(1):249-64.

38. Weiss JM, Andersson PL, Lamoree MH, Leonards PE, van Leeuwen SP, Hamers T. Competitive binding of poly- and perfluorinated compounds to the thyroid hormone transport protein transthyretin. Toxicol Sci. 2009;109(2):206-16.

39. Haddow JE, McClain MR, Lambert-Messerlian G, Palomaki GE, Canick JA, Cleary-Goldman J, Malone FD, Porter TF, Nyberg DA, Bernstein P, et al. Variability in thyroid-stimulating hormone suppression by human chorionic [corrected] gonadotropin during early pregnancy. J Clin Endocrinol Metab. 2008;93(9):3341-7.

40. Glinoer D, de Nayer P, Bourdoux P, Lemone M, Robyn C, van Steirteghem A, Kinthaert J, Lejeune B. Regulation of maternal thyroid during pregnancy. J Clin Endocrinol Metab. 1990;71(2):276-87.

41. Okada E, Kashino I, Matsuura H, Sasaki S, Miyashita C, Yamamoto J, Ikeno T, Ito YM, Matsumura T, Tamakoshi A, et al. Temporal trends of perfluoroalkyl acids in plasma samples of pregnant women in Hokkaido, Japan, 2003-2011. Environ Int. 2013;60:89-96.

42. Miyashita C, Sasaki S, Saijo Y, Okada E, Kobayashi S, Baba T, Kajiwara J, Todaka T, Iwasaki Y, Nakazawa H, et al. Demographic, behavioral, dietary, and socioeconomic characteristics related to persistent organic pollutants and mercury levels in pregnant women in Japan. Chemosphere. 2015;133:13-21. 